\title{
Bargetz, Brigitte: Ambivalenzen des Alltags. Neuorientierungen für eine Theorie des Politischen, 296 S., transcript, Bielefeld 2016.
}

\section{Franziska Martinsen}

Online publiziert: 23. Januar 2020

(C) Der/die Autor(en) 2020

In ihrer Studie über den Alltag als ,politischen Kampfplatz“ (S. 34) beschäftigt sich Brigitte Bargetz mit zwei zunächst gegensätzlich erscheinenden Bereichen menschlichen Lebens, die jedoch, und dies legt sie überzeugend dar, einander bedingen und im glücklichsten Fall (im Anschluss an Autor_innen wie Karl Marx, Paul Ricœur, Henry Lefebvre, Agnes Heller und Audre Lorde) eine wechselseitige, emanzipativ-transformative Wirkung innerhalb der Gesellschaft entfalten können. Die Rede ist hier zum einen vom Profan-unauffälligen, Selbstverständlichsten: dem Alltag, und zum anderen vom Spezifisch-wissenschaftlichen, normativ Hochaufgeladenen: von der Theoretisierung des Politischen. Bargetz zeigt, dass ausgehend von Alltäglichkeit, Alltagspraxis und -wissen die Grundlagen politischer Theoretisierung neu entwickelt werden können. Hierbei geht sie von drei Prämissen aus: Erstens versteht die Autorin die politische Begriffs- und Theoriebildung als Ausdruck und zugleich als Reflexions- und Bearbeitungsform politischer Verhältnisse. Zweitens liegt der Untersuchung ein wissenschaftsgeschichtlich tradiertes Verständnis von Alltag zugrunde, nach dem die Auseinandersetzung mit Alltag stets als Intervention in denselbigen zu begreifen sei. Die dritte Prämisse konkretisiert die zweite: Alltag, als Intervention verstanden, stellt dann nicht einfach einen deskriptiv zu erfassenden Untersuchungsgegenstand dar, sondern ein wissenschaftliches Konzept, mit dem Grundzüge einer kritischen politischen Theorie des Alltags ermittelt werden (vgl. Kap. I.5).

In fünf Teile gliedert sich das Buch. Auf den einleitenden Abschnitt (Kap. I) folgt ein Teil (Kap. II), der im Sinne einer Kartografie die Koordinaten einer Theorie des Politischen erläutert, der zufolge Politik mehr als Staat, Staatlichkeit und institutionalisierte Politik umfasst und nicht auf die rationalen Interessen von Akteur_innen beschränkt ist. Vielmehr wird sie im Rekurs unter anderem auf Chantal Mouffe als agonistisch-demokratische, gesellschaftliche Praxis verstanden. Komplementär dazu widmet sich der dritte Teil der Erläuterung des Alltagskonzepts, wie es für die Studie relevant ist, nämlich nicht als empirisch zu beschreibendes Phänomen, sondern als aus der feministischen Kritik an der hierarchischen Überordnung des

F. Martinsen $(\bowtie)$

Bremen, Deutschland

E-Mail: f.martinsen@ipw.uni-hannover.de 
Politischen über das Private entwickelte, kritisch begriffene Denkfigur (Kap. III). Es schließen sich wiederum zwei einander ergänzende Teile IV und V an, die als Hauptstück des Buches zu lesen sind. Hier plausibilisiert Bargetz ihre These, dass Elemente einer kritischen Theorie im Wechselverhältnis von Alltag und Politik zu entwickeln seien: Während Teil IV den Alltag politiktheoretisch reflektiert, entfaltet Teil V die Theorie des Politischen alltagstheoretisch. In diesem Zusammenhang wendet sich die Verfasserin der Rolle von Gefühlen, Affekten und Emotionen zu, die traditionell in der Politiktheorie dem Bereich des ,Privaten' zugeordnet und dem als rational konnotierten Bereich des ,Politischen` entgegengesetzt wurden (vgl. insbesondere V.3.3). Die Untersuchung hat kein eigentliches Abschlusskapitel, sondern endet etwas abrupt mit einem kurzen Ausblick darauf, wie das alltägliche Gefühl der Wut, zum Beispiel über erfahrene Ungerechtigkeit, als Motor für Politik fungieren kann. Angesichts der in den letzten Jahren vielerorts auftretenden selbsterklärten Wutbürger_innen erhält dieses nicht weiter ausgeführte, letzte Kapitel (V.4) nun eine Brisanz, die sich als aktueller denn je erweist. Bargetz' Überlegungen verweisen darauf, wie dringlich die Auseinandersetzung mit der politischen Bedeutung des Alltags ist. Denn nicht erst im Akt der Politisierung selbst tritt die politische Relevanz des Alltäglichen zutage. Eine politische Theorie des Alltags hat laut Bargetz die Ambivalenz der Verstrickung von Rationalität und Emotionalität nicht deshalb zu berücksichtigen, damit Alltag ,enttrivialisiert ' und ihm eine höhere Weihe verliehen werden könne. Ihr Anliegen lässt sich vielmehr so interpretieren, dass nur eine alltagsbezogene Theorie des Politischen den ebenso banalsten wie kompliziertesten Tatbestand zu erfassen vermag: Dass die politische Gestaltung der Gesellschaft (zumindest in einer Demokratie) alle angeht und eben nicht von den wenigen Experten oder Berufspolitiker_innen, sondern buchstäblich von allen zu leisten ist.

Demgegenüber ließe sich einwenden, dass die ,Neuorientierung' der politischen Theorie etwas vollmundig daherkommt, pocht doch der Feminismus längst darauf, dass das Private politisch sei. Tatsächlich gelingt Brigitte Bargetz aber eine originäre ,Entdeckung ‘ des Alltags, und der Erkenntnisgewinn ihrer Studie liegt genau darin, dass mit „Alltag“ die übliche Dichotomie von ,privat“ und ,öffentlich“ aufgelöst wird und die mit ihr verbundenen exkludierenden Zuweisungen aufgebrochen werden können. Denn es ist eben jene empirische Einsicht, die die Autorin überzeugend für die politische Theorie fruchtbar zu machen vermag: Von der politischen Dimension des Alltags sind alle betroffen, die nicht Tier oder Gott sind, wie es schon bei Aristoteles heißt. Die Wahrnehmung von politischen Anliegen und Problemen wird von der alltäglichen Erfahrung innerhalb von Gesellschaften geprägt und sie ist aufgrund ihrer Alltäglichkeit prinzipiell jedem Menschen zugänglich. Dass dieses Alltagswissen nicht per se als emanzipatorisch zu fassen ist, verleugnet Bargetz dabei nicht, auch wenn für sie das emanzipative Potenzial für die eigenen Überlegungen insbesondere im Gefolge der eingangs aufgezählten Autor_innen im Vordergrund steht. Schließlich geht es ihr nicht um eine einseitige Affirmation des Alltags, sondern um die kritische politische Theorie des Alltaglebens als „Theorie des Möglichen“ (S. 103).

Funding Open Access funding provided by Projekt DEAL. 
Open Access Dieser Artikel wird unter der Creative Commons Namensnennung 4.0 International Lizenz veröffentlicht, welche die Nutzung, Vervielfältigung, Bearbeitung, Verbreitung und Wiedergabe in jeglichem Medium und Format erlaubt, sofern Sie den/die ursprünglichen Autor(en) und die Quelle ordnungsgemäß nennen, einen Link zur Creative Commons Lizenz beifügen und angeben, ob Änderungen vorgenommen wurden.

Die in diesem Artikel enthaltenen Bilder und sonstiges Drittmaterial unterliegen ebenfalls der genannten Creative Commons Lizenz, sofern sich aus der Abbildungslegende nichts anderes ergibt. Sofern das betreffende Material nicht unter der genannten Creative Commons Lizenz steht und die betreffende Handlung nicht nach gesetzlichen Vorschriften erlaubt ist, ist für die oben aufgeführten Weiterverwendungen des Materials die Einwilligung des jeweiligen Rechteinhabers einzuholen.

Weitere Details zur Lizenz entnehmen Sie bitte der Lizenzinformation auf http://creativecommons.org/ licenses/by/4.0/deed.de.

\section{Best, Heinrich/Higley, John (Hrsg.): The Palgrave Handbook of Political Elites, 698 S., Palgrave Macmillan, London 2018.}

\section{Sylvia Veit}

Online publiziert: 16. Januar 2020

(c) Gesellschaft zur wissenschaftlichen Förderung politischer Literatur e.V. and the Author(s) 2020

Heinrich Best (Friedrich-Schiller-Universität Jena) und John Higley (University of Connecticut) haben mit dem knapp 700 Seiten umfassenden Handbuch eine bemerkenswerte Bestandsaufnahme der Forschung zu politischen Eliten vorgelegt. Beide Herausgeber haben sich über mehrere Jahrzehnte hinweg mit diesem Thema beschäftigt. Politische Eliten sind dabei nicht in einem engen Sinne ausschließlich als politische Entscheidungsträger zu verstehen, sondern werden weiter definiert als alle Personen, die Einfluss auf politische Entscheidungen ausüben. $\mathrm{Zu}$ ihnen gehören somit neben den bekannten Machteliten (Wirtschaft, Regierung, Militär) auch Personen, die wichtige Positionen in Parteien und Parlamenten, großen Interessenorganisationen und Verbänden, bedeutenden Medienunternehmen oder der Kirche innehaben. Im Einzelfall können dies auch Personen sein, die formal keine herausgehobenen Positionen innehaben, die aber dennoch großen Einfluss ausüben. Wesentlich ist, dass es sich gesamtgesellschaftlich gesehen um eine relativ kleine,

S. Veit $(\bowtie)$

Kassel, Deutschland

E-Mail: sveit@uni-kassel.de 